\title{
Template for Reporting Results of Biomarker Testing of Specimens From Patients With Myeloproliferative Neoplasms
}

\author{
Todd W. Kelley, MD, Daniel A. Arber, MD, Christine Gibson, CTR, Daniel Jones, MD, PhD, \\ Joseph D. Khoury, MD, Bruno C. Medeiros, MD, Dennis P. O'Malley, MD, Keyur P. Patel, MD, \\ PhD, Monika Pilichowska, MD, Mohammad A. Vasef, MD, Jeremy Wallentine, MD, James L. \\ Zehnder, MD, and for the Members of the Cancer Biomarker Reporting Committee, College \\ of American Pathologists \\ Department of Pathology, University of Utah, Salt Lake City (Dr Kelley); the Departments of \\ Pathology (Drs Arber and Zehnder) and Medicine (Dr Medeiros), Stanford University School of \\ Medicine, Stanford, California; the Blood and Marrow Transplantation Program, Moffitt Cancer \\ Center, Tampa, Florida (Ms Gibson); the Department of Pathology, Ohio State University, \\ Columbus (Dr Jones); the Department of Hematopathology, Division of Pathology and Laboratory \\ Medicine, The University of Texas MD Anderson Cancer Center, Houston (Drs Khoury and Patel); \\ the Department of Pathology, Clarient Pathology Services, Aliso Viejo, California (Dr O'Malley); \\ the Department of Pathology and Laboratory Medicine, Tufts Medical Center, Boston, \\ Massachusetts (Dr Pilichowska); the Department of Pathology, University of New Mexico Health \\ Sciences Center, Albuquerque (Dr Vasef); and the Department of Pathology, Intermountain \\ Healthcare, Salt Lake City, Utah (Dr Wallentine)
}

\begin{abstract}
The College of American Pathologists (CAP) offers these templates to assist pathologists in providing clinically useful and relevant information when reporting results of biomarker testing. The CAP regards the reporting elements in the templates as important elements of the biomarker test report, but the manner in which these elements are reported is at the discretion of each specific pathologist, taking into account clinician preferences, institutional policies, and individual practice.
\end{abstract}

The CAP developed these templates as educational tools to assist pathologists in the useful reporting of relevant information. It did not issue them for use in litigation, reimbursement, or other contexts. Nevertheless, the CAP recognizes that the templates might be used by hospitals, attorneys, payers, and others. The CAP cautions that use of the templates other than for their intended educational purpose may involve additional considerations that are beyond the scope of this document.

The inclusion of a product name or service in a CAP publication should not be construed as an endorsement of such product or service, nor is failure to include the name of a product or service to be construed as disapproval.

Reprints: Todd W. Kelley, MD, Department of Pathology, University of Utah, 15 N Medical Dr East, Rm 2100, Salt Lake City, UT 84112 (todd.kelley@ path.utah.edu).

The authors have no relevant financial interest in the products or companies described in this article. 


\section{Template for Reporting Results of Biomarker testing of Specimens from Patients with Myeloproliferative Neoplasms}

Completion of the template is the responsibility of the laboratory performing the biomarker testing and/or providing the interpretation. When both testing and interpretation are performed elsewhere (eg, a reference laboratory), synoptic reporting of the results by the laboratory submitting the tissue for testing is also encouraged to ensure that all information is included in the patient's medical record and thus readily available to the treating clinical team.

\section{Biomarker Reporting Template \\ Myeloproliferative Neoplasms (MPNs) \\ Select a single response unless otherwise indicated-Note: Use of this template is optional.* \\ *Reporting on the data elements in this template is not required.}

\section{Specimen Type}

Peripheral blood

Bone marrow

Isolated granulocytes from peripheral blood

Other (specify):

\section{Results}

Cytogenetic Testing Results (Karyotype)

No abnormalities detected

__ Abnormal karyotype detected

(specify):

Fluorescence In Situ Hybridization Testing

$B C R-A B L 1$

No $B C R-A B L 1$ fusion detected

$B C R-A B L 1$ fusion detected (specify percentage of positive cells): $\%$ PDGFRA

No PDGFRA fusion detected

FIP1L1-PDGFRA fusion detected (specify percentage of positive cells):

$\%$ 
Other PDGFRA fusion detected (specify percentage of positive cells):

$\%$

PDGFRB

No PDGFRB fusion detected

ETV6-PDGFRB fusion detected (specify percentage of positive cells): $\%$

Other $P D G F R B$ fusion detected (specify percentage of positive cells): $\%$

FGFR1

No FGFR1 rearrangement detected

FGFR 1 rearrangement detected (specify percentage of positive cells): $\%$

BCR-ABL1 Transcript Reverse Transcription-Polymerase Chain Reaction (RT-PCR) Testing

No $B C R-A B L 1$ fusions detected

$B C R-A B L 1$ fusions detected

If quantitative testing performed:

$B C R-A B L 1$ normalized copy number ( $B C R-A B L 1 /$ reference gene):

Percent $B C R-A B L 1$ on International Scale (e13/14a2 (p210) fusions only): $\%$

\section{JAK2 p.V617F (c. 1849G.T) Mutation Testing}

No mutation detected

Mutation detected

For $J A K 2 \mathrm{p} . \mathrm{V} 617 \mathrm{~F}$, if test is quantitative, specify quantitative value:

Reported as:

_ Percent mutant allele burden

Percent transcript levels

__ Normalized copy number (V617F transcripts/ reference gene)

\section{Additional Mutation Testing}

$J A K 2$ exon 12

No $J A K 2$ exon 12 mutation detected 
$J A K 2$ exon 12 mutation detected (specify mutation):

MPL

No MPL mutation detected

MPL mutation detected (specify mutation):

$C A L R$ (calreticulin)

No $C A L R$ mutation detected

$C A L R$ mutation detected (specify mutation):

$K I T$

No KIT mutation detected

KIT mutation detected (specify mutation):

___ Other (specify gene):

No mutation detected

Mutation detected (specify mutation):

\section{Methods}

BCR-ABL1 Transcript RT-PCR Testing

Assay sensitivity:

\section{JAK2 p.V617F (c. 1849G.T) Mutation Testing}

Assay sensitivity:

Assay method:

___ Allele-specific PCR

__ Sanger sequencing

__ Pyrosequencing

__ Next-generation sequencing

Other (specify):

\section{Other Mutation Testing (Specify Gene):}

Assay sensitivity:

Assay method: 
_Allele-specific PCR

_ Sanger sequencing

Pyrosequencing

Next-generation sequencing

Other (specify):

Exon(s)/codon(s) covered:

\section{Explanatory Notes}

Myeloproliferative neoplasms are clonal disorders characterized by the expansion of one or more myeloid lineages, leading to increased bone marrow cellularity and elevated peripheral blood myeloid cell counts. The latter may manifest as granulocytosis, erythrocytosis, thrombocytosis, or a combination of these, depending on the disease subtype. The diagnosis and classification of MPNs require synthesis of the clinical, morphologic,

immunophenotypic, and molecular genetic findings. During the course of the last few years, the spectrum of genetic mutations identified in MPNs has expanded, and PCR and/or sequence-based mutation testing is now routinely incorporated into the diagnostic workup. However, the diagnosis still relies heavily on the peripheral blood and bone marrow morphologic findings and the clinical features of the disease, particularly for those patients who do not have a disease-defining genetic abnormality.

In the 2008 World Health Organization classification system, the category of MPNs includes chronic myelogenous leukemia; chronic neutrophilic leukemia; polycythemia vera; primary myelofibrosis; essential thrombocythemia; chronic eosinophilic leukemia; mastocytosis; and MPN, unclassifiable; and the clinical and pathologic findings may overlap with the category of myeloid and lymphoid neoplasms with eosinophilia and abnormalities of PDGFRA, $P D G F R B$, and FGFR $1 .{ }^{1}$ Classical cytogenetic karyotyping and fluorescence in situ hybridization testing are often used in the evaluation of patients to test for the presence of $\mathrm{t}(9 ; 22)(\mathrm{q} 34 ; \mathrm{q} 11.2) ; B C R-A B L 1$ - particularly for those who present with neutrophilic leukocytosis - and for abnormalities of PDGFRA, PDGFRB, and FGFR 1 for those patients who present with eosinophilia. Otherwise, patients with MPNs may have a variety of cytogenetic abnormalities. Various trisomies, such as +8 and/or +9 , are often identified. Given the degree of standardization and specialization that has occurred in $B C R-A B L 1$ testing, and the repeated nature of the analyses, CAP has published a separate chronic myelogenous leukemia monitoring template for those patients known to have chronic myelogenous leukemia.

When the cytogenetic and/or fluorescence in situ hybridization testing results are nonspecific or negative, it may be necessary to use additional molecular genetic tests. The $J A K 2$ p.V617F (c.1849G.T) somatic point mutation is present in almost all patients with polycythemia vera and in a large proportion (40\%-50\%) of patients with essential thrombocythemia or primary myelofibrosis. Both qualitative and quantitative testing methods are employed, although the utility of quantitation of the mutant $J A K 2$ allele burden remains somewhat controversial. A small percentage of patients with polycythemia vera who 
lack evidence of a $J A K 2$ p.V617F mutation may have a mutation in exon 12 of $J A K 2$, and these are often insertions or deletions. ${ }^{2}$ Different testing methods are often used for $J A K 2$ p.V617F and $J A K 2$ exon 12 mutations, and it should be noted that different methods-for example, Sanger sequencing and allele-specific PCR - may have markedly different sensitivities. Mutations in the $C A L R$ (calreticulin) gene were recently identified in most patients with essential thrombocythemia or primary myelofibrosis who lack $J A K 2$ mutations. ${ }^{3,4}$ Less commonly, mutations in the MPL gene are present in a subset of essential thrombocythemia/primary myelofibrosis patients without $J A K 2$ or $C A L R$ mutations. ${ }^{3}$ KIT mutation testing is helpful for the diagnosis and subclassification of mastocytosis and is important for determining the likely response to tyrosine kinase inhibitor therapy. ${ }^{5}$

Given the pace of recent findings, additional pathologically relevant mutations are likely to be identified and/or clinically validated in the near future. With this in mind, the template includes space for reporting other mutation testing, and future template updates will reflect additional molecular genetic findings that may be incorporated into the World Health Organization classification system.

\section{References}

1. Swerdlow, SH., Campo, E., Harris, L., et al. World Health Organization Classification of Tumours. 4th. Vol. 2. Lyon, France: IARC Press; 2008. World Health Organization Classification of Tumours of Hematopoietic and Lymphoid Tissues.

2. Scott LM, Tong W, Levine RL, et al. JAK2 exon 12 mutations in polycythemia vera and idiopathic erythrocytosis. New Engl J Med. 2007; 356(5):459-468. [PubMed: 17267906]

3. Klampfl T, Gisslinger H, Harutyunyan AS, et al. Somatic mutations of calreticulin in myeloproliferative neoplasms. New Engl J Med. 2013; 369(25):2379-2390. [PubMed: 24325356]

4. Nangalia J, Massie CE, Baxter EJ, et al. Somatic mutations of calreticulin in myeloproliferative neoplasms. New Engl J Med. 2013; 369(25):2391-2405. [PubMed: 24325359]

5. Ma Y, Zeng S, Metcalfe DD, et al. The c-KIT mutation causing human mastocytosis is resistant to STI571 and other KIT kinase inhibitors; kinases with enzymatic site mutations show different inhibitor sensitivity profiles than wild-type kinases and those with regulatory-type mutations. Blood. 2002; 99(5):1741-1744. [PubMed: 11861291] 